\title{
A Voltage Controlled Oscillator with Inductive Divider Design and Analysis at Frequencies Above $100 \mathrm{GHz}$
}

\author{
Yasir Shafiullah \\ Centre for Wireless Communications \\ University of Oulu \\ Oulu, Finland \\ yasir.shafiullah@oulu.fi
}

\author{
Rehman Akbar \\ Centre for Wireless Communications \\ University of Oulu \\ Oulu, Finland \\ rehman.akbar@oulu.fi \\ Aarno Pärssinen \\ Centre for Wireless Communications \\ University of Oulu \\ Oulu, Finland \\ aarno.parssinen@oulu.fi
}

\author{
Mikko Hietanen \\ Centre for Wireless Communications \\ University of Oulu \\ Oulu, Finland \\ mikko.hietanen@oulu.fi
}

\begin{abstract}
Millimeter wave oscillators are of particular interest towards future communications and sensing as frequency multiplication of local oscillator signal is resulting in degraded performance. At high frequencies, the quality (Q) factor of the LC tank is degraded. Thus, a higher transconductance ( $\left.G_{M}\right)$ size is required to compensate the losses, thereby consuming high power. Numerous techniques have been adopted to reduce the power consumption of a cross-coupled LC voltage controlled oscillator ( $\mathrm{VCO}$ ) and to improve the negative resistance $\left(R_{n e g}\right)$ associated to it. One of them utilizes inductors in the cross-coupled pair. In this paper, we examine the tradeoffs of this inductor to improve negative resistance above $\mathbf{1 0 0}$ GHz. For the analysis, the transistor model is being verified with the simplified small-signal equivalent model. Moreover, we have studied the inductor influence on the transition frequencies of the cross-coupled pair. The restrictions of this technique at high frequencies is investigated. To study the benefits of this technique, an oscillator core is designed and simulated using the $22 \mathrm{~nm}$ FDSOI CMOS process. It exhibits a tuning range of $\mathbf{5 . 4 \%}$ from $125.5 \mathrm{GHz}$ to $132.5 \mathrm{GHz}$, and phase noise from -101.23 $\mathrm{dBc} / \mathrm{Hz}$ to $-102.0381 \mathrm{dBc} / \mathrm{Hz}$ at $10 \mathrm{MHz}$ offset over the entire tuning range. The VCO consumes power of $17 \mathrm{~mW}$. In simulations, an improvement in power consumption by $4.3 \mathrm{~mW}$ and phase noise by $1.5 \mathrm{dBc} / \mathrm{Hz}$ were achieved when compared to conventional LC Tank VCO.
\end{abstract}

Keywords-VCO, CMOS, LC-VCO, inductive divider, tuning range, phase noise, sub-terahertz, voltage-controlled oscillators, mm-wave, radio frequency.

\section{INTRODUCTION}

Conventional cross-coupled pair VCOs at mm-wave frequencies require large transconductance $\left(\mathrm{G}_{\mathrm{M}}\right)$ to compensate for the losses incurred by the LC tank due to the low quality factor $(\mathrm{Q})$ of the varactors or capacitors. The $\mathrm{Q}$ value affects the phase noise (PN) performance whereas the contribution of the fixed parasitic capacitance $\left(\mathrm{C}_{\mathrm{par}}\right)$ by the active circuitry in the VCO affects the frequency tuning range (FTR), respectively. Moreover, having a large $\mathrm{G}_{M}$ in the circuit is power-hungry and consumes a lot of power to generate negative resistance. Thus, low power, highperformance VCO design is of essence. In the past decade, efforts have been made to design a low voltage, low power i.e. mm-wave CMOS VCO. An effective impedance balancing technique was introduced in [1] by placing an inductor between the gate and drain terminals of the negative $\mathrm{G}_{\mathrm{M}}$ crosscoupled pair stage as shown in Fig. 1 (b). The inductor assisted in the improvement of the negative resistance and therefore the size of the $\mathrm{G}_{\mathrm{M}}$ transistors in the cross-coupled pair stage was reduced improving power consumption, phase noise and tuning range.

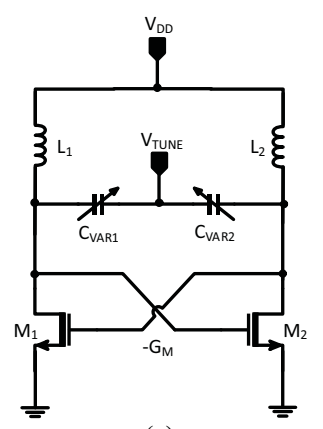

(a)

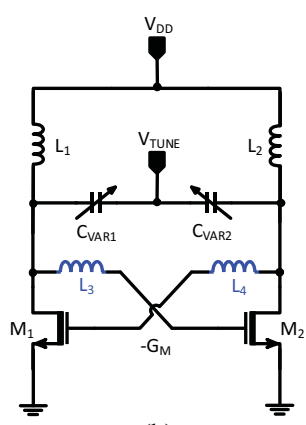

(b)
Fig. 1. (a) Conventional cross-coupled LC oscillator (b) An Inductive divider cross-coupled LC oscillator.

In [1], the designed oscillator achieved a tuning range of $8.75 \mathrm{GHz}$ at a center frequency of $64 \mathrm{GHz}$, consuming 3.16 $\mathrm{mW}$ power when operating at a $\mathrm{V}_{\mathrm{DD}}$ of $0.6 \mathrm{~V}$. The same technique was further excelled in the designing of a D-Band VCO operating at $118 \mathrm{GHz}$ with a $7.8 \%$ tuning range consuming $5.6 \mathrm{~mW}$ [2]. The method is quite useful in the design of a low power mm-wave VCO with improvements in phase noise performance and tuning range. Although the technique is convenient, the possible drawbacks of this technique at high frequencies above $100 \mathrm{GHz}$ hasn't been discussed in the references.

In this paper, we address the limitations and performance challenges in the design and implementation of the inductive divider VCO above $100 \mathrm{GHz}$. An in-depth analysis is performed to explore the feasibility of this technique to achieve high operational frequency. The paper is organized as follows. In Section II, conventional LC VCO and inductive divider VCO are briefly discussed. The impact of the $\mathrm{Q}$ factor, parallel resistive losses $\left(\mathrm{R}_{\mathrm{p}}\right)$ at high frequencies are examined. In Section III the inductive divider cross-coupled pair is compared with its small signal equivalent model. The negative resistance enhancement parameters and the constraints limiting the performance are discussed in detail. This section also explains the practical implementation of the circuit design and the layout. Simulation results are shown in Section IV, and finally Section V concludes the paper. 


\section{DESIGN CONSIDERATION FOR High-SPEED VCO}

\section{A. Fundamental LC cross-coupled pair topology}

The fundamental LC tank cross-coupled VCO is shown in Fig. 1 (a). It consists of two parts, an LC tank, and negative $\mathrm{G}_{\mathrm{M}}$ cross-coupled pair. The LC tank acts as a resonator and has losses associated with it. To compensate for the losses, a negative resistance is required. Cross-coupled transistors have an inherited property to generate negative resistance. However, the transistors are usually large and consume a lot of power to generate sufficiently large negative resistance. Designing a fundamental VCO in the high frequency, i.e., DBand, is a challenge in terms of the performance and trade-off with the tuning range, phase noise, and power consumption need to be balanced against specifications.

\section{B. Quality Factor of the LC tank}

The quality factor of the LC tank in the VCO is highly degraded when operating at $\mathrm{mm}$-wave and higher frequencies. The inherently low $\mathrm{Q}$ of the varactors degrades the tank $\mathrm{Q}$, consequently affecting the phase noise performance as predicted by the Leeson phase noise model [3]. At high frequencies, the varactor governs the quality factor of the tank. The $\mathrm{Q}$ of the LC tank is given as

$$
\frac{1}{Q_{\text {tank }}}=\frac{1}{Q_{\text {ind }}}+\frac{1}{Q_{\text {var }}},
$$

where $\mathrm{Q}_{\text {tank }}$ is the quality factor of the tank, $\mathrm{Q}_{\text {ind }}$ is the quality factor of the inductor and $\mathrm{Q}_{\mathrm{var}}$ is the quality factor of the varactor.

The varactor $\mathrm{Q}$ degradation over the frequency range is shown in Fig. 2 (a). In order not to use large varactors to avoid $\mathrm{Q}$ factor issues, one would inevitably compromise the tuning range achievable in the design. Due to the compromise between phase noise and the tuning range, an optimum balance is essential to achieve higher oscillation frequencies.

To target higher oscillation frequencies, the size of the inductor or varactor in the LC tank is reduced, consequently reducing the parallel resistive losses associated with the tank that is represented by $R_{p}$ as illustrated in Fig. 2 (b). There is a requirement to have an equal or higher negative resistance $R_{\text {neg }}$ to compensate for the losses produced by the tank. To satisfy the oscillation condition, the $\left|R_{\text {neg }}\right|$ should be equal or smaller than $R_{p}$. An example to estimate the parallel resistance $\left(R_{p}\right)$ over the frequency range at different inductance $(\mathrm{L})$ values with the varactor capacitance $\left(\mathrm{C}_{\mathrm{var}}\right)$ kept constant at $24 \mathrm{fF}$ is shown in Fig. 2 (b). The anticipated $R_{p}$ is around 170-230 $\Omega$. The oscillation frequency is calculated simply as

$$
f_{\text {osc }}=\frac{1}{2 \pi \sqrt{L C_{v a r}}}
$$

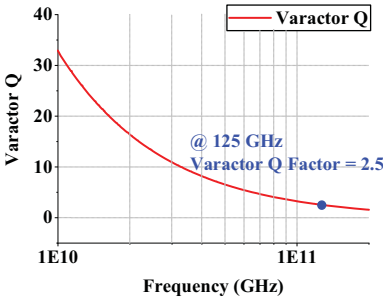

(a)

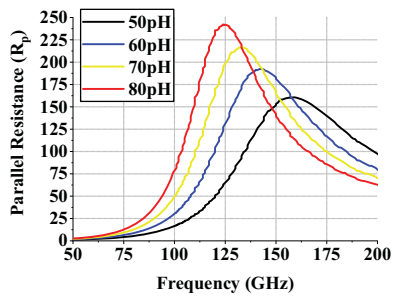

(b)
Fig. 2. (a) Quality factor of the varactor over the frequency (b) Parallel resistance $R_{p}$ at different values of inductance $L$ in the $L C$ tank.

\section{Fundamental LC tank inductive divider cross-coupled pair topology}

The inductive divider topology is slightly different from the conventional cross-coupled pair topology, as shown in Fig. 1 (b). Since, the transistors in the $\mathrm{G}_{\mathrm{M}}$ cross-coupled pair are responsible for producing the negative resistance, an addition of passive element in the cross-coupled pair, i.e., inductors $\mathrm{L}_{3}$ and $\mathrm{L}_{4}$ will assist in boosting the negative resistance provided by these transistors. Eventually, the size of the $\mathrm{G}_{\mathrm{M}}$ transistors will reduce lowering the parasitic capacitance $C_{p a r}$ of the $G_{M}$ cross-coupled pair transistors. The power utilized by these transistors is reduced with improvement in the oscillation frequency $\mathrm{f}_{\mathrm{osc}}$ of the VCO. In practical design, the oscillation frequency $\mathrm{f}_{\text {osc }}$ takes into account the total inductance $\mathrm{L}_{\mathrm{T}}$ and varactor capacitance $\mathrm{C}_{\mathrm{var}}$ of the $\mathrm{LC}$ tank, the parasitic capacitance $\mathrm{C}_{\mathrm{par}}$ of the $\mathrm{G}_{\mathrm{M}}$ cross-coupled pair transistors and the loaded buffer capacitance $\mathrm{C}_{\text {buff }}$ is given as:

$$
f_{\text {osc }}=\frac{1}{2 \pi \sqrt{L_{T}\left(C_{\text {var }}+C_{\text {par }}+\left(2 C_{\text {buff }}\right)\right)}} .
$$

\section{NEGATIVE RESISTANCE ENHANCEMENT TECHNIQUES, MODELING, LIMITATIONS AND IMPLEMENTATION}

The negative resistance $R_{\text {neg }}$ provided by the conventional $\mathrm{G}_{\mathrm{M}}$ cross-coupled pair, as shown in Fig. 1 (a), is approximately $-2 / g_{m}$ where $g_{m}$ is the transconductance of the individual transistor. Inherently, the gate and the drain impedances of the transistors $\mathrm{M}_{1}$ and $\mathrm{M}_{2}$, are mismatched. The introduction of the inductor in cross-coupled pair in Fig. 3 (a) assists in the conjugate matching of the drain and the gate impedances, thus improving the power efficiency [1]. Moreover, the technique helps in enhancing the negative resistance generated by the cross-coupled pair, which can be shown from the differential small-signal equivalent model of the inductor in the cross-coupled pair stage as in Fig. 3 (b). The estimated real part of the input impedance looking into the cross-coupled pair is given as

$$
Z_{\text {in(real })} \approx \frac{-2}{g_{m}}+\frac{2 \omega^{2} L_{g} C_{g s}}{g_{m}},
$$

where $\mathrm{g}_{\mathrm{m}}$ is the transconductance of the transistor, $\mathrm{C}_{\mathrm{gs}}$ is the gate-to-source capacitance, $\mathrm{L}_{\mathrm{g}}$ is the inductor in the crosscoupled pair and $\omega$ is the required oscillation frequency.

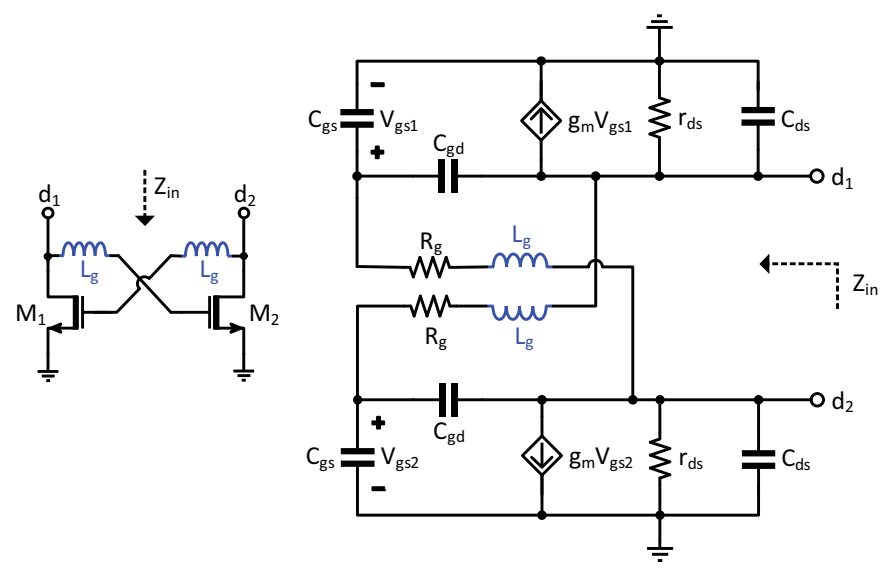

(a)

(b)

Fig. 3. (a) Transistor Model (b) Small signal equivalent model. 
The real part of the impedance of the conventional crosscoupled pair is modified with this technique. The real impedance has a second term, which is a function of the inductor $\mathrm{L}_{\mathrm{g}}$, frequency $\omega$ and the gate-to-source capacitance $\mathrm{C}_{\mathrm{gs}}$. The parasitic capacitances of the transistors are assumed to be fixed. Theoretically, the parameters that can assist in the improvement of the negative resistance $R_{n e g}$ are an increase of inductance value $\mathrm{L}_{\mathrm{g}}$ and operational frequency $\omega$. An increase of $18 \%$ is expected in the negative resistance theoretically at $130 \mathrm{GHz}$ with an $\mathrm{L}_{\mathrm{g}}$ value of $15 \mathrm{pH}$ in Eq. (4).

\section{A. Constraints and Limitations}

The behavior of the negative resistance can be extracted from the simplified small-signal equivalent model presented in Fig. 3 (b). Extracted parameters are compared with the process design kit (PDK) transistor model. The comparison of the behavior of the negative resistance $R_{\text {neg }}$ over the frequency is shown in Fig. 4 at a fixed $\mathrm{L}_{\mathrm{g}}$ value of $15 \mathrm{pH}$. Both models are in agreement with each other. At low frequencies, the negative resistance is approximately equal to $-2 / \mathrm{g}_{\mathrm{m}}$. At high frequencies, there is a transition in the negative resistance $R_{\text {neg, }}$, and it flips from the negative to positive. This transition is defined as the "maximum attainable oscillation frequency" for the cross-coupled negative resistance cell originally proposed by [4]. This behavior is primarily due to gate resistance $r_{g}$. The impedances offered by gate-to-source capacitance $\mathrm{C}_{\mathrm{gs}}$ becomes comparable to the gate resistance $r_{g}$ at high frequencies, and a large portion of the voltage at gate appears across $r_{g}$ rather than $C_{g s}$ leading to inversion. It is worth noting that as the transition frequency approaches, $\left|R_{\text {neg }}\right|$ drops rapidly, thus making the value of $\left|R_{\text {neg }}\right|$ even larger than $R_{p}$. Therefore, stable oscillation condition cannot be satisfied by limiting the performance at high frequencies. The transition frequency $\omega_{\text {trans }}[5]$ for the conventional cross-coupled negative resistance cell is a function of $r_{g}$ and $C_{g s}$ and is given as

$$
\omega_{\text {trans }} \approx \sqrt{\frac{g m}{2 \pi\left(C_{g s}+C_{g d}\right) r_{g} C_{g s}}},
$$

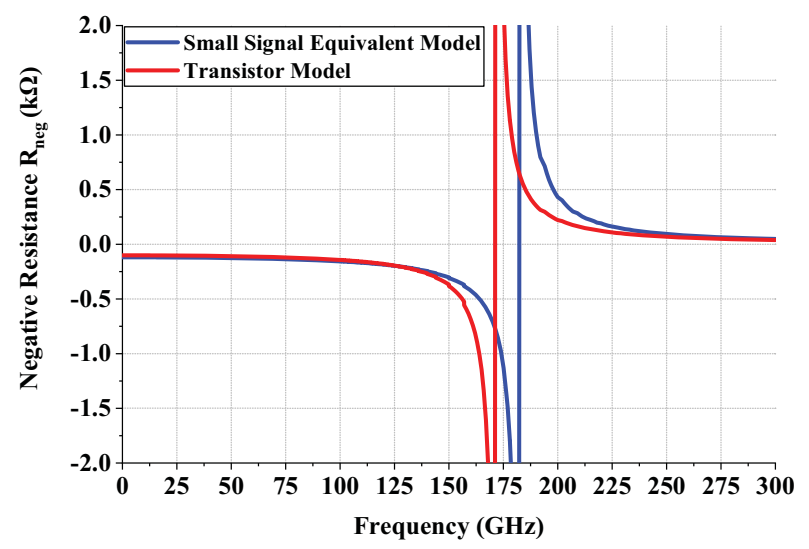

Fig. 4. Negative resistance $R_{\text {neg }}$ variation vs. frequency (Transistor Model vs. Small signal equivalent model).

The inductor $\mathrm{L}_{\mathrm{g}}$ in cross-coupled pair, as expressed in Eq. (4), has the ability to improve the negative resistance but suffers at high frequencies. This behavior is simulated in the small signal equivalent model by observing the negative resistance over the variation of the inductance $L_{g}$ at different frequencies from 60 to $140 \mathrm{GHz}$ and is depicted in Fig. 5 . There is an improvement in the negative resistance $R_{\text {neg. The }}$ decrease in value of negative resistance $\mathrm{R}_{\text {neg }}$ in the beginning of the curve at each frequency iteration indicates the transition frequency approaching quickly. The variation in the improvement of negative resistance is significantly reduced at a higher frequency, and the negative resistance curve is almost flattened at $140 \mathrm{GHz}$ inductance value of $25 \mathrm{pH}$. Beyond 25 $\mathrm{pH}$ no improvement can be seen. The flattening of the curve at high frequencies demonstrates the limitation of this technique to enhance the negative resistance. To further investigate this, behavior in frequency domain at different inductance $\mathrm{L}_{\mathrm{g}}$ values is shown in Fig. 6. It can be seen that the increase in inductance value from 5 to $30 \mathrm{pH}$ shifts the transition frequency from $190 \mathrm{GHz}$ to $160 \mathrm{GHz}$, indicating that $\mathrm{Lg}_{\mathrm{g}}$ is a direct function of the transition frequency $\omega_{\text {trans }}$ along with $r_{g}$ and $C_{g s}$ expressed in Eq. (5). Therefore, higher the value of $\mathrm{Lg}_{\mathrm{g}}$ earlier the transition frequency occurs thus limiting the maximum oscillation frequency. Hence, this justifies the curve flattening behavior at $140 \mathrm{GHz}$ in Fig. 5 with no major improvement in the negative resistance. Layout optimization techniques can be utilized to minimize the dependence of the transition frequency $\omega_{\text {trans }}$ on $r_{g}$ and $\mathrm{C}_{\mathrm{gs}}$. The appropriate frequency to practice this technique is to make use of the improvement in negative resistance before the curve flattening occurs. For instance in Fig. 5, at $140 \mathrm{GHz}$ the inductance values below $25 \mathrm{pH}$ can be utilized. It is to be noted that there is a slight parasitic capacitance contribution by the inductor $\mathrm{L}_{\mathrm{g}}$ itself which is lower compared the $\mathrm{R}_{\text {neg }}$ it generates [2].

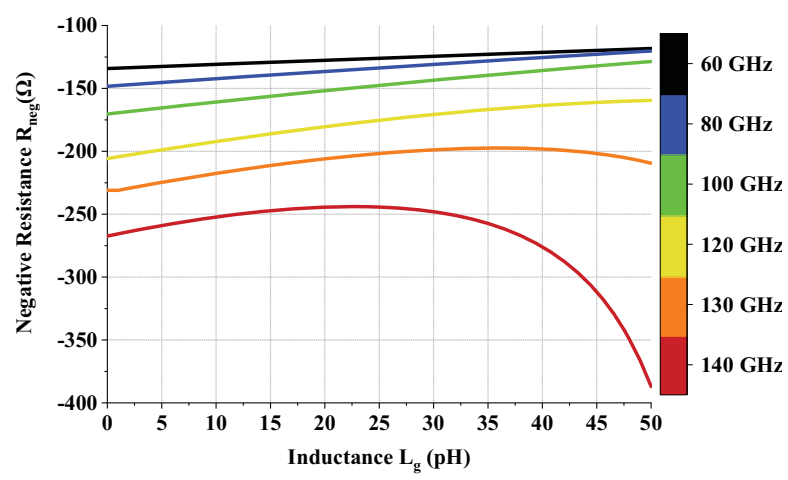

Fig. 5. Negative resistance $R_{\text {neg }}$ variation vs. inductance $L_{g}$ modeled at different frequencies using the small signal equivalent model.

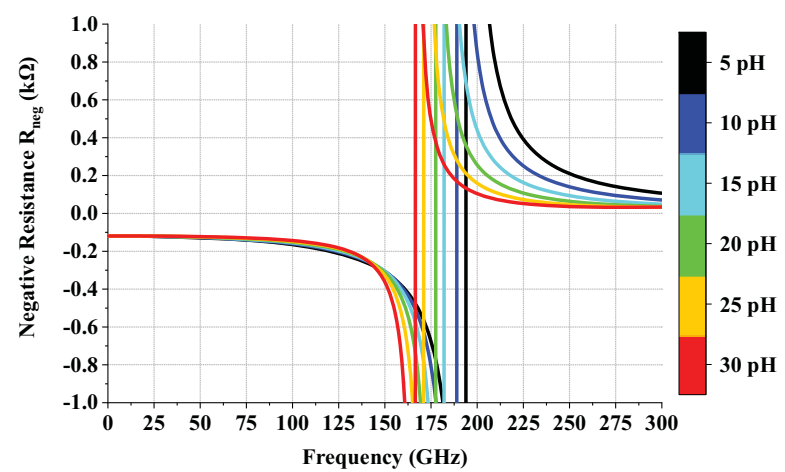

Fig. 6. Negative resistance $R_{\text {neg }}$ variation vs. frequency modeled at different inductance $\mathrm{L}_{\mathrm{g}}$ values using the small signal equivalent model.

\section{B. Practical implementation aspects}

The practical implementation of the inductive divider cross-coupled pair suffers from the performance trade-offs at higher frequencies, as discussed in the previous section. The transistors from the PDK were utilized. An ideal inductor in the cross-coupled pair was used to analyze the behavior. The 
performance variation of the negative resistance $R_{\text {neg }}$ and parasitic capacitance $\mathrm{C}_{\mathrm{par}}$ versus the variation of inductor $\mathrm{L}_{\mathrm{g}}$ from 1 to $30 \mathrm{pH}$ is investigated in Fig. 7 at different frequencies from 65 to $150 \mathrm{GHz}$. The negative resistance $\mathrm{R}_{\text {neg }}$ curve changes its behavior with the increase in frequency. As the frequency increases, the variation in the increase in negative resistance tends to decline as shown in Fig. 7 (a-d) curve flattens at $150 \mathrm{GHz}$. After $130 \mathrm{GHz}$, there is hardly any improvement, and a severe decline is observed in the negative resistance. This uncertain behavior with the increase in frequency represents the limitation discussed earlier.

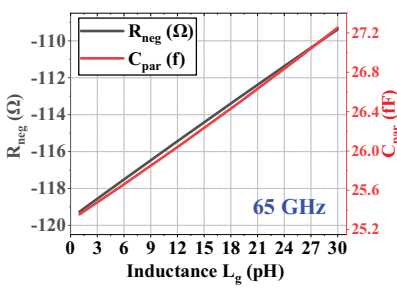

(a)

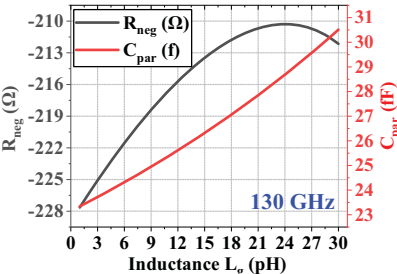

(c)

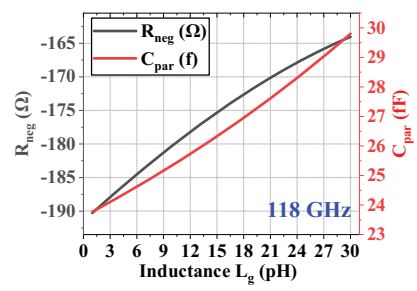

(b)

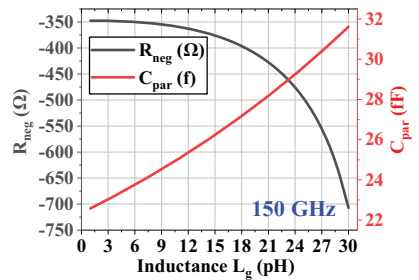

(d)
Fig. 7. Negative Resistance $\left(R_{n e g}\right)$ and parasitic capacitance $\left(C_{p a r}\right)$ versus inductance $\mathrm{L}_{\mathrm{g}}$

\section{Layout Implementation}

The layout of the oscillator core is realized in a $22 \mathrm{~nm}$ FDSOI process. The inductor $\mathrm{L}_{\mathrm{g}}$ is implemented by the transmission line which is connected to the gate and drain of the transistors in the cross-coupled pair. The negative resistance is extracted by varying the transmission line length, as shown in Fig. 8 which varies the inductance consequently. The variation of the negative resistance is quite low. The inductor in the LC tank is a single turn differential inductor and is utilizing the ultra-thick metal layer. The $\mathrm{Q}$ of the inductor is a function of frequency, and high $\mathrm{Q}$ is expected from the inductor at high frequencies. The inductor selfresonance frequency and losses were taken into consideration in the design process. Varactor at high frequencies suffers from a low Q. The varactor was selected to have a decent capacitance tuning range and a reasonable quality factor. The varactor is placed in between the two terminals of the inductor. The layout arrangement is shown in Fig. 9. The nominal supply voltage is $0.8 \mathrm{~V}$.

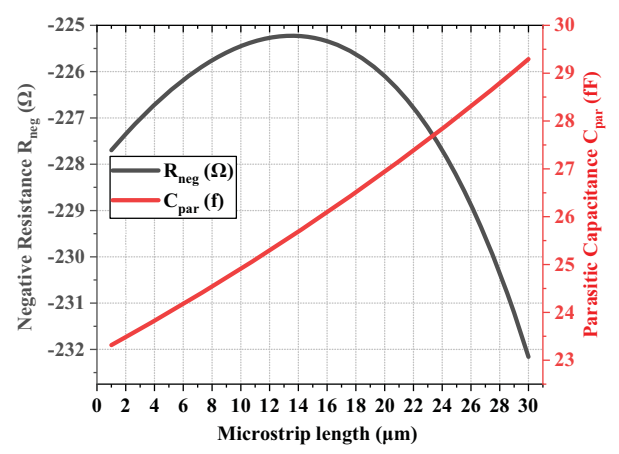

Fig. 8. At $130 \mathrm{GHz}\left(\mathrm{R}_{\text {neg }}\right.$ and $\mathrm{C}_{\mathrm{par}}$ versus Transmission line length $\left.(\mu \mathrm{m})\right)$
The simulated oscillator core consumes a DC power of $17.1 \mathrm{~mW}$. It oscillates at a center frequency of $129 \mathrm{GHz}$ with a phase noise of $-100.6 \mathrm{dBc} / \mathrm{Hz}$. The tuning range of the oscillator core is observed to be $5.4 \%$, with the tuning voltage range from 0 to $0.8 \mathrm{~V}$. The output signal has a voltage swing of $640 \mathrm{mV}_{\mathrm{p}-\mathrm{p}}$ to $1.6 \mathrm{k} \Omega$ load. It is worth noting that output buffers are not included in the circuitry. The buffer circuitry will further reduce the tuning range and the oscillation frequency due to the contribution of the loaded capacitance.

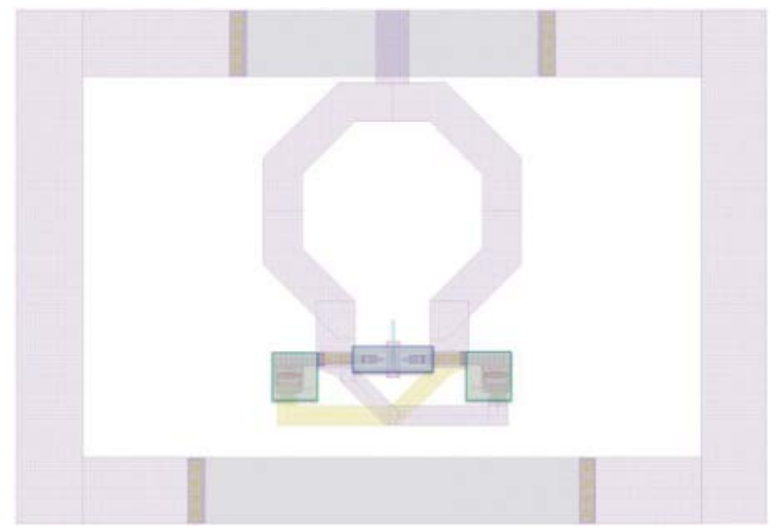

Fig. 9. Inductive divider LC VCO core layout.

\section{SimULATION RESULtS}

Table I presents a performance summary of the simulated design of the VCO with and without the inductor $\mathrm{L}_{\mathrm{g}}$ in the cross-coupled pair. In comparison the inductive divider has a degradation of $4 \mathrm{GHz}$ in the oscillation frequency and 1\% decrease in tuning range as compared to the cross-coupled VCO. The degradation reflects the limitations associated with this implementation. However, the inductive divider technique showed considerable improvement of $1.5 \mathrm{dBc} / \mathrm{Hz}$ in phase noise and a $4.3 \mathrm{~mW}$ improvement in DC power consumption. In Table I, results are also compared to the stateof-the-art oscillator designs at $64 \mathrm{GHz}$ and $118 \mathrm{GHz}$ utilizing a similar inductive divider concept. The VCOs designed with this technique are power efficient with an improvement in phase noise performance.

TABLE I. PERFORMANCE SUMMARY AND COMPARISONS WITH THE STATE OF THE ART

\begin{tabular}{|c|c|c|c|c|c|}
\hline \multirow{2}{*}{ Reference } & \multirow{2}{*}{$\begin{array}{c}{[1]} \\
90 n m\end{array}$} & \multirow{2}{*}{\multicolumn{2}{|c|}{$\frac{[2]}{65 \mathrm{~nm}}$}} & \multirow{2}{*}{\multicolumn{2}{|c|}{$\begin{array}{c}\text { This work* } \\
22 n m\end{array}$}} \\
\hline & & & & & \\
\hline Topology & $\begin{array}{c}\text { Ind. } \\
\text { divider } \\
\text { LC type }\end{array}$ & $\begin{array}{c}\text { Ind. } \\
\text { divider } \\
\text { LC type }\end{array}$ & $\begin{array}{c}\text { Ind. } \\
\text { divider } \\
\text { LC type }\end{array}$ & $\begin{array}{l}\text { Fund. } \\
\text { LC }\end{array}$ & $\begin{array}{c}\text { Ind. } \\
\text { divider } \\
\text { LC type }\end{array}$ \\
\hline $\mathrm{f}_{\mathrm{osc}}(\mathrm{GHz})$ & 64 & 118.3 & 122.5 & 133 & 129 \\
\hline $\mathrm{V}_{\mathrm{DD}}$ & 0.6 & 1 & 0.8 & 0.8 & 0.8 \\
\hline $\mathrm{P}_{\mathrm{DC}}(\mathrm{mW})$ & 3.16 & 5.6 & 2 & 21.4 & 17.1 \\
\hline FTR (\%) & 8.75 & 4.4 & 7.8 & 6.54 & 5.4 \\
\hline $\begin{array}{c}\text { Phase } \\
\text { Noise } \\
(\mathrm{dBc} / \mathrm{Hz})\end{array}$ & -95 & -83.9 & -83 & -99.1 & -100.6 \\
\hline $\begin{array}{l}\text { Phase } \\
\text { Noise } \\
\text { Offset } \\
(\mathrm{MHz})\end{array}$ & 1 & 1 & 1 & 10 & 10 \\
\hline Description & $\begin{array}{l}\text { Core }+ \\
\text { Buffer }\end{array}$ & $\begin{array}{l}\text { Core }+ \\
\text { Buffer }\end{array}$ & $\begin{array}{l}\text { Core }+ \\
\text { Buffer }\end{array}$ & Core & Core \\
\hline $\mathrm{FOM}_{\mathrm{T}}$ & -185 & -175.7 & -174.6 & -176.2 & -176.53 \\
\hline
\end{tabular}




\section{CONCLUSION}

Here, we presented the limitations and bottlenecks in the design of a D-Band inductive divider LC VCO. The technique is useful in the design of low power VCOs utilizing power matching technique by applying the inductor in the crosscoupled pair. The performance of this technique is compromised at the D-Band frequencies and beyond. The aid in the transition frequency by the addition of the inductor in the $\mathrm{G}_{\mathrm{M}}$ cross-coupled pair stage limits the performance of the $\mathrm{VCO}$ at these frequencies. The variation in the improvement of the negative resistance decreases as the operational frequency approaches the transition frequency. The prototype VCO core utilizing the technique was designed using a $22 \mathrm{~nm}$ FDSOI process. The VCO was able to achieve a decent output power and a low power consumption of $17 \mathrm{~mW}$ with a slight improvement in phase noise. The analyzed technique illustrated is a good choice in the design of low power VCO while taking into consideration the performance constraints involved.

\section{ACKNOWLEDGMENT}

This research has been financially supported by the Academy of Finland 6Genesis Flagship (grant 318927). The authors would like to thank Global Foundries for support and providing PDK.

\section{References}

[1] L. Li, P. Reynaert, and M. Steyaert, "A low power mm-wave oscillator using power matching techniques," in Digest of Papers - IEEE Radio Frequency Integrated Circuits Symposium, 2009.

[2] W. Volkaerts, M. Steyaert, and P. Reynaert, " $118 \mathrm{GHz}$ fundamental VCO with $7.8 \%$ tuning range in $65 \mathrm{~nm}$ CMOS," in Digest of Papers - IEEE Radio Frequency Integrated Circuits Symposium, 2011.

[3] D. B. Leeson, "A Simple Model of Feedback Oscillator Noise Spectrum," Proc. IEEE, 1966.

[4] H. Veenstra and E. Van Der Heijden, "A 19-23 GHz integrated LC-VCO in a production $70 \mathrm{GHz}$ fT SiGe technology," in European Solid-State Circuits Conference, 2003.

[5] B. Jung and R. Harjani, "High-frequency LC VCO design using capacitive degeneration," in IEEE Journal of Solid-State Circuits, 2004. 